${ }^{1}$ Department of Endodontics, Endodontics Specialization Career, School of Dentistry, University of Buenos Aires (UBA), Buenos Aires, Argentina.

${ }^{2}$ Department of Histology and Embryology, School of Dentistry, University of Buenos Aires (UBA), Buenos Aires, Argentina

${ }^{3}$ Institute of Cell Biology and Neurosciences "Prof. E. de Robertis "(IBCN) - School of Medicine, University of Buenos Aires (UBA) - National Research Council (CONICET), Buenos Aires, Argentina.
Corresponding author:

Mariela C. Canzobre

Marcelo T. de Alvear 2142 (1-A),

Buenos Aires, Argentina

+5491151076672

mariela.canzobre@odontologia.uba.ar

Editor: Dr Altair A. Del Bel Cury

Received: December 19, 2020

Accepted: May 25, 2021

\section{Regenerative endodontics model in immature infected rat molars using two step protocol}

\author{
Liliana Sierra $^{1}$ iD , Ángeles Castrillo ${ }^{1}$ iD, Elizabeth \\ Ritacco ${ }^{1}$, Haydeé Miño Cornell ${ }^{1}$, Mariela C Canzobre ${ }^{2,3_{,}^{*}}$ (iD)
}

Aim: to develop a model for regenerative endodontics using newly-weaned Wistar rats immature molars with pulp necrosis to histologically describe the evolution of apical tissues following treatment with a bi-antibiotic paste, induced bloodclot formation and MTA. Methods: Ten 25-day-old female Wistar rats were divided into an initial control group (Ci) and two experimental groups in which pulp necrosis was experimentally induced on the left mandibular first molar by exposing the pulp chamber and leaving it open to the oral environment. One of the experimental groups was left untreated (E1) while the other was submitted to a protocol of regenerative endodontics 10 days thereafter (E2). Fifteen days after placement of a bi-antibiotic paste, bleeding was induced into the root canal space and MTA was placed upon. Animals were euthanized 30 days later. Right mandibular first molars served as an 80-day-old final control group (Cf). Each hemimandible was histologically processed to analyse parameters associated with root development. Statistical analysis was carried by means of ANOVA; $p$ values below 0.05 were considered statistically significant. Results: baseline (i.e. 25-days old) mean root length and apical diameter of the distal root canal were $1.84 \pm 0.25$ and $0.38 \pm 0.02 \mathrm{~mm}$ respectively. Following the regenerative endodontic protocol, cells lining the walls of the root canal and significant increase to both length $(2.37 \pm 0.22 \mathrm{~mm})$ and diameter $(0.32 \pm 0.03 \mathrm{~mm})$ were observed. Conclusions: newly-weaned Wistar rats serve as a suitable model to evaluate regenerative endodontic protocols. However, further research is needed in order to disclose the nature of the cells and/or cell mediators involved.

Keywords: Regenerative endodontics. Dental pulp necrosis. Root canal therapy. Anti-bacterial agents. 


\section{Introduction}

The American Association of Endodontists has defined regenerative endodontic therapy as "biologically based procedures designed to replace damaged structures, including dentine and root structures, as well as cells of the pulp-dentine complex"1. Continued root development, tooth survival and function retention have been long pursued; from the very first studies carried on humans and dogs by Ostby ${ }^{2}$ in 1961, when a new apical tissue was observed after the formation of an intracanal blood clot, to the more recent advances in tissue engineering. Considering the core principles of tissue engineering, - a triad that includes an appropriate source of stem/progenitor cells, growth factors, and scaffolds - regenerating functioning pulp tissue would be the ideal therapeutic approach when treating immature teeth with open apexes and a diagnosis of pulp necrosis ${ }^{3,4}$.

Immature open apexes are richly vascularised and some cells of the apical papilla and Hertwig's Epithelial Root Sheath (HERS) might be able to survive infection ${ }^{5}$. Consequently, if noxious stimuli are removed, these cells become able to proliferate in proximity to the periapical tissues leading to the regeneration of radicular and periradicular tissues such as alveolar bone, periodontal ligament, cementum and dentine-pulp complex thereby allowing the root to mature increasing both in length and thickness ${ }^{6}$. A number of clinical studies have reported positive pulpal testing results after complete maturation of the root ${ }^{7}$. Notwithstanding, the nature of the tissues regenerated within the root canal space is still unknown and under investigation. Considering human samples are difficult to harvest, animal models might contribute to shedding some light on this matter and identifying cell populations as well as the intrinsic and extrinsic factors that might participate in the process of apexogenesis/maturogenesis ${ }^{6,8,9}$.

A paradigm shift in Endodontics currently presents us with the alternative to perform a protocol based in endodontic regenerative procedures (REPS) when treating an immature permanent tooth with an open apex and diagnosis of pulp necrosis: to induce, by generation an intracanal blood clot, the formation of new tissues that can assist continued root maturation. When unsuccessful, apexification protocols always remain an option ${ }^{10}$.

Developing a standardised protocol using laboratory animals and materials such as bi-antibiotic paste and Mineral Trioxide Aggregate (MTA) that permits the study of the histologic characteristics of the newly formed tissues within the canal and in the periapical region remains a challenge $\mathrm{e}^{11-13}$

The aim of the present study was to develop a model for regenerative endodontics using newly-weaned Wistar rat immature molars with pulp necrosis by means of a bi-antibiotic paste followed by evoked bleeding and formation of a blood clot within the root canal and its contact with a biomaterial. Moreover, we sought to assess the progress of root maturation in length and diameter of the root canal following treatment and describe the histology of the apical and periapical tissues

\section{Materials and Methods}

This study was approved by "Ethics Committee of the School of Dentistry of the University of Buenos Aires (CD N012/2016 CICUAL)" 
Ten 25-day-old female Wistar rats were used. Animals were housed in cages in groups of five animals per cage maximum within the animal facility of the Department of Histology and Embryology of the School of Dentistry of the University of Buenos Aires under standard controlled conditions (temperature $18-22^{\circ} \mathrm{C}$, relative humidity $40-60 \%$ and 12:12 light/dark cycles - approximately 100 lux -) and were allowed ad libitum access to food (standard chow, Cooperación, Buenos Aires, Argentina) and water. Both, animals and environmental conditions, were monitored daily to guarantee they remained stable throughout the entire study.

Newly-weaned rats (i.e. 25-day-old) animals were randomly allocated to one of the following groups:

Control group ( $\mathrm{Ci} ; n=3$ ): animals weighing about $60 \mathrm{~g}$ average were used as an initial control group to study the histological characteristics of the mandibular first molar of the rat molar at the age of 25 days.

Experimental group $1(E 1 ; n=3)$ : pulp necrosis was experimentally induced on the left mandibular first molar as explained below and received no further treatment.

Experimental group $2(E 2 ; n=4)$ : following experimentally-induced pulp necrosis, a protocol of regenerative endodontics was performed.

Experimental induction of pulp necrosis: animals in the experimental groups were anaesthetised with a combination of $50 \mathrm{mg} / \mathrm{kg}$ of ketamine (Ketamid; Holliday-Scott S.A., Beccar, Buenos Aires, Argentina) and $10 \mathrm{mg} / \mathrm{kg}$ of xylazine (Kensol; König S.A., Avellaneda, Buenos Aires, Argentina) administered i.p. Once anaesthetised, each animal was laid in a dorsal position on a stretcher immobilising its head by the upper incisors, which are placed through a metal wire attached to the headrest. Mouth opening, tongue separation and mandibular first molar isolation were guaranteed by an aluminium clamp as proposed by a protocol presented recently ${ }^{14}$.

In order to achieve complete necrosis of the dental pulp, the pulp chamber was accessed by drilling through its roof using a size 1/4 round dental bur (SS White, Lakewood, New Jersey) mounted on an electric-engine driven micromotor and left open to the oral environment for 10 days.

Regenerative endodontic therapy protocol: left mandibular first molars of animals allocated to the E2 group were further treated following the protocol previously described by Thibodeau and Trope ${ }^{15}$. Ten days after pulp exposure, the pulp chamber was cleaned using a dentine excavator and a $2.5 \%$ sodium hypochlorite solution; the distal canal was catheterised using a \#08 K-file (Dentsply Sirona, Ballaigues, Switzerland) calibrated at a working length of $3 \mathrm{~mm}$ and slowly irrigated with a $2.5 \%$ sodium hypochlorite (Clorox, Argentina) solution followed by saline; no further mechanical preparation was performed. The root canal was then dried using sterile paper points \#25 (Meta Biomed; Cheongju, North Chungcheong, South Korea) and filled with a bi-antibiotic paste containing equal amounts of $200 \mathrm{mg}$ ciprofloxacin and $500 \mathrm{mg}$ metronidazole (2Mix) mixed with propylene glycol ${ }^{15}$. The access cavity was sealed with glass-ionomer cement (Ketac Molar EasyMix, 3M ESPE, Neuss, Germany).

Fifteen days after intervention, the pulp chamber of the left mandibular first molar was accessed again and cleaned using a dentine excavator. The bi-antibiotic paste was 
gently flushed out of the canal with $2.5 \%$ sodium hypoclorite (Clorox, Argentina). After final irrigation with 17\% EDTA (Tedequim, Argentina) for 5 minutes, the canal was dried using sterile paper points (Meta Biomed; Cheongju, North Chungcheong, South Korea). A \#15 K-file (Dentsply Sirona, Ballaigues, Switzerland) was inserted through the distal canal beyond the foramen to induce bleeding from the periapical tissues ${ }^{16}$ and formation of a blood clot inside the root canal. MTA was then placed upon the distal root canal entrance orifice and compacted using a wet cotton pellet to assist its setting reaction; the access cavity was finally sealed with glass-ionomer cement (Ketac Molar EasyMix, 3M ESPE, Neuss, Germany). Coronal seals were daily monitored for wear derived from rodents' diet so as to prevent their loss and root canal reinfection.

Animals were euthanized by i.p. injection of a lethal dose of pentobarbital sodium (Euthanyle, Brouwer S.A., Buenos Aires, Argentina) 30 days after placement of MTA (E2). Experimental group E1 which received no further treatment was matched in age by the time of euthanasia, set to take place at approximately the same time of the day in order to minimise the effect of changes owed to circadian rhythm. All right mandibular first molars were kept as an 80 day-old control group (Cf) (Fig. 1).

A

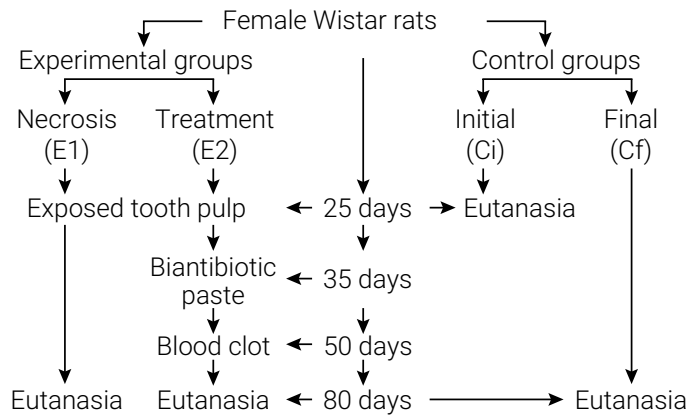

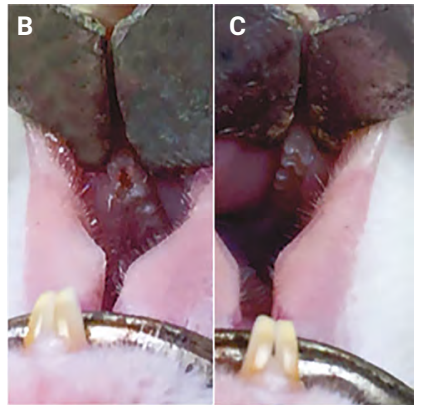

Figure 1. The experimental protocol and the procedures carried upon both experimental and control groups are shown in A. Photographs depict the isolation of the mandibular first molar with its pulp exposed (B) and the intact mandibular first molar that served as control (C).

In order to perform bright-field microscopy evaluation, right and left hemimandibles were dissected and fixed in buffered formalin ( $\mathrm{pH}$ 7.3-7.5) for 48 hs. Following decalcification in EDTA 10\% pH 7 for 5 weeks, hemimandibles were dehydrated and embedded in paraffin (Paraplast, Sigma-Aldrich) and serial mesio-distally oriented sections of the mandibular first molars of approximately $7 \mu \mathrm{m}$ were obtained using a Minot microtome. Sections were stained with hematoxylin and eosin $(\mathrm{H}-\mathrm{E})$ and digitalised microphotographs were then taken with a light microscope (Nikon Eclipse Ni, Nikon Instruments Inc, Melville, NY, USA) and analysed using ImagePro Plus4.5.0.29 software to measure (Fig. 2) the length and apical diameter of the distal root canal as described in previous studies ${ }^{14}$. In order to do this, one line was drawn passing through the mesial and distal cement enamel junctions (anatomic limit between the crown and root surface) and another one tangent to the apex of the mesial and distal roots. With these as references, two lines were drawn: a perpendicular one running through the centre of the distal canal (a: root canal length) and a parallel one at the 
level of the apical third of the root from inner dentine surface located on the distal side to the one on the mesial side (b: apical diameter of the distal root canal). Furthermore, we measured the size of the periapical area as enclosed by the line tangent to the apex of the distal root and the surface of the alveolar bone proper as well as the osteoclastic cells within this area considering all cells close to the bone surface and containing two or more nuclei (Fig. 2).

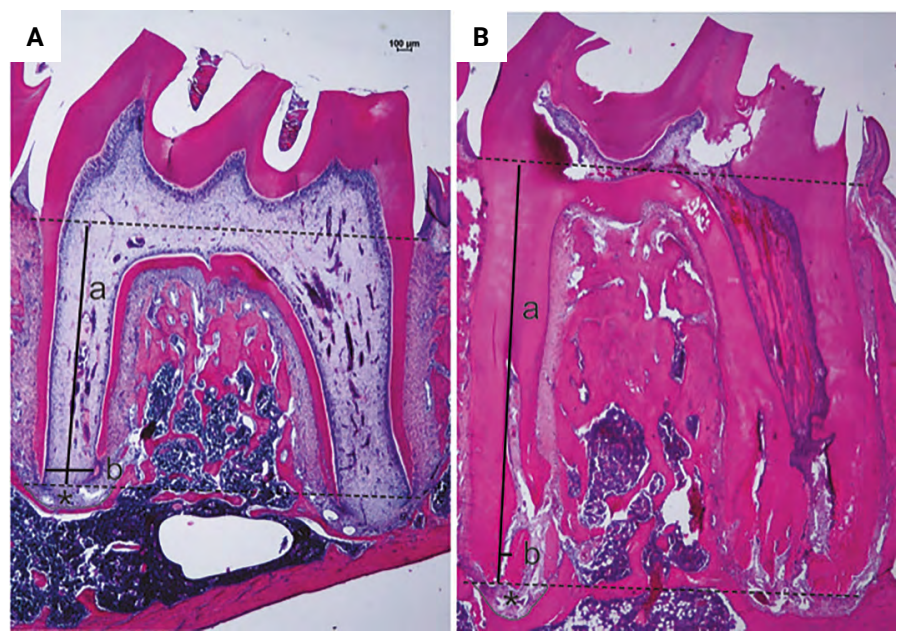

Figure 2. Microphotographs showing HE stained histological sections of the mandibular first molar corresponding to the initial (A; i.e. 25-day-old) and final (B; i.e. 80-day-old) control groups. Dashed lines served as landmarks passing through the cement enamel junction and the apex of both roots. Lines $a$ and $b$ were drawn to measure the length of the distal root and the apical diameter of its root canal, respectively. The asterisk indicates the location of the periapical area. Note that, A presents a wide root canal as well as very thin walls and HERS can still be devised.

\section{Statistical analysis}

Graph Pad Prism 5.0 was employed. All the results were expressed as mean and standard deviation (SD), and statistically analysed using ANOVA followed by Tukey's posthoc test setting the significance level at $5 \%$.

\section{Results}

\section{Histomorphometric analysis of root development}

As observed on the $\mathrm{H}$-E stained histological sections, molars of the 25-day-old control group (Ci) exhibited thin root canal walls as well as wide root canals, pulp with a normal histological pattern and wide open apexes where HERS could still be observed (Fig 2A). Mean values for root canal length and apical diameter of the canal of the distal root of the mandibular first molar are shown in Table 1; statistically significant differences were found between Ci and E2 ( $p<0.05)$ and between $\mathrm{Ci}$ and $\mathrm{Cf}(\mathrm{p}<0.001)$. With regards to the periapical area, this group showed a normal width without signs of inflammation. A high number of osteoclasts was observed, as expected for a developing alveolus which is being remodelled constantly in order to accommodate a maturing root. 
Table 1. Mean values and standard deviations of the distal root length and canal diameter, size of the periapical area and osteoclastic activity.

\begin{tabular}{lcccc}
\hline \multicolumn{5}{c}{ Wistar rat mandibular first molar } \\
\hline Distal root & $\begin{array}{c}\text { Ci: Control } \\
\text { (25 days old })\end{array}$ & $\begin{array}{c}\text { E1: Pulp } \\
\text { necrosis }\end{array}$ & E2: REPs & $\begin{array}{c}\text { Cf: Control } \\
\text { (80 days old })\end{array}$ \\
\hline Length $(\mathrm{mm})$ & $1.84 \pm 0.25^{\mathrm{ab}}$ & $1.65 \pm 0.16^{\mathrm{cd}}$ & $2.37 \pm 0.22^{\text {ace }}$ & $3.20 \pm 0.13^{\text {bde }}$ \\
\hline Apical canal diameter $(\mathrm{mm})$ & $0.38 \pm 0.02^{\mathrm{ab}}$ & $0.39 \pm 0.02^{\text {cd }}$ & $0.32 \pm 0.03^{\text {ace }}$ & $0.08 \pm 0.01^{\text {bde }}$ \\
\hline Periapical area $\left(\mathrm{mm}^{2}\right)$ & $0.19 \pm 0.02^{\mathrm{ab}}$ & $1.23 \pm 0.34^{\mathrm{a}}$ & $0.88 \pm 0.46^{\mathrm{bc}}$ & $0.14 \pm 0.05^{\mathrm{c}}$ \\
\hline Osteoclasts $/ \mathrm{mm}$ & $1.79 \pm 0.55^{\mathrm{a}}$ & $1.72 \pm 0.45^{\mathrm{b}}$ & $1.19 \pm 0.23$ & $0.36 \pm 0.42^{\text {ab }}$ \\
\hline
\end{tabular}

Values sharing the same letter within a row show a statistical difference between groups (ANOVA $p<0.05)$

Group E1 (80 days old) revealed that pulps exposed to the oral environment and left untreated until euthanasia were entirely necrotic; these molars exhibited no sign of HERS and very thin root canal walls. A greater width of the periapical periodontal ligament space was observed (Ci vs E1 p<0.001) at expense of the presence of an inflammatory infiltrate and resorption of the surrounding alveolar bone by osteoclasts (E1 vs Cf $\mathrm{p}<0.001$ ). However, no odontoclasts were found (Fig. 3) which translated into a lack of statistically significant differences when comparing root length and apical diameter of the root canal to the Ci group (Table 1).

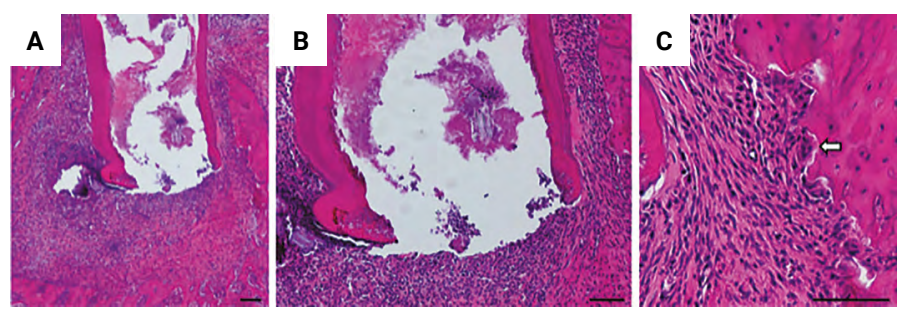

Figure 3. Microphotographs showing HE stained histological sections of the experimental distal root of the mandibular first molar (E1) with pulp necrosis and immature apex (A). Necrotic tissue can be seen inside the root canal; neither odontoblasts lining the dentine surface or HERS can be observed (B). Actively resorbing osteoclasts and Howship lacunae can be seen on the alveolar bone surface (arrow in $\mathrm{C}$ ). Bars represent $100 \mu \mathrm{m}$.

Group E2 (80 days old), in which the distal root of the left mandibular first molar was treated by means of a regenerative protocol, revealed an increase in root length and a decrease in the width of the root canal and its apical diameter as well as slightly thicker root canal walls (E2 vs E1 p<0.05 and E2 vs Cf p<0.001) (Table 1). The width of the periapical periodontal ligament space was significantly greater than that of the control groups (Ci vs E2 p<0.05; Cf vs E2 $p<0.05$ ) but a reduction in the inflammatory infiltrate, size of the periapical area and the number of osteoclasts were found when compared with E1 (no significant difference). Cells lining the dentinal surface of the root canal and osteoblasts on the alveolar bone surface could be observed (Fig. 4). 


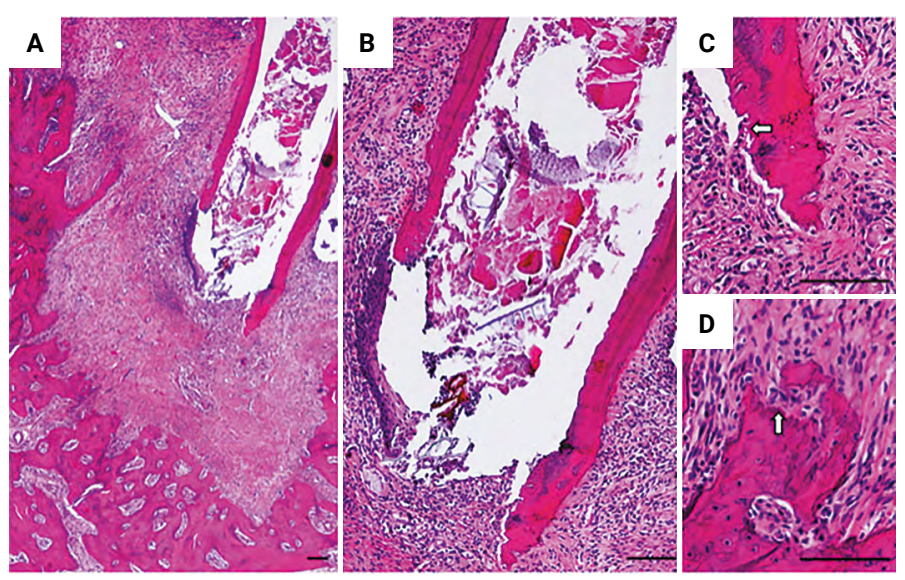

Figure 4. Microphotographs showing HE stained histological sections of the experimental distal root of the left mandibular first molar (E2) that received regenerative endodontic therapy (A). Blood cells and MTA particles can be seen inside the root canal (B). Although there is no evidence of HERS, some cells can be seen lining the root canal walls (arrow in C). Active osteoblasts and a thin layer of osteoid can be seen on the alveolar bone surface (arrow in D). Bars represent $100 \mu \mathrm{m}$.

In spite of the improved root development, the length of the distal root in the group that received the regenerative endodontic protocol did not get to match the degree of maturation of the right mandibular first molar (Cf) used as an 80 day-old control given the latter measured $3.2 \pm 0.12 \mathrm{~mm}$, presented thick dentinal walls and a completely developed apex narrowed by the continuous deposition of cellular cementum (hypercementosis). In this group, lateral canals were also evident probably as a consequence of the rapid deposition of cellular cementum in relation to blood vessels. The periapical area exhibited normal width and histological characteristics and an alveolar wall with little cell activity (Fig 2B and Table 1).

\section{Discussion}

Stem cell research brought along new therapeutic approaches to treat immature permanent teeth with a diagnosis of pulp necrosis. REPs constitute a promising alternative but the results derived from different protocols, whether they be currently approved or under investigation, require experimental studies to guarantee more predictable patient outcomes in the future.

Several biological aspects related to tissue regeneration and repair are still missing likely due to the ethical and casuistic limitations associated with human trials. For this reason, animals such as dogs, cats, monkeys, rabbits, ferrets and rats are used by different groups in the search for an experimental model in vivo that would prove to be suitable and reproducible in order to study an analyse regenerative processes ${ }^{12}$. The animal models of REPs currently available in the literature ${ }^{11-13,15,17}$ do not adhere to the three stages as presented in clinical protocols. For example, Scarparo et al. ${ }^{17}$ also used necrotic immature teeth but, unlike our model, they only performed root canal disinfection with no further evoked bleeding and blood clot formation. Previous reports using animal models have more commonly used a triple antibiotic paste to achieve disinfection of the root canal|1,13,17; we, however, opted for a bi-antibiotic paste 
thus ruling minocycline out, as suggested by Thibodeau and Trope ${ }^{15}$ in 2007, thereby implementing a protocol that is presently considered more appropriate in a clinical setting as minocycline is associated with dental discoloration.

We used Wistar rats as they can be easily housed, anaesthetised and handled, and enable histological sections to be obtained relatively fast, at a lower cost of acquisition and maintenance with less concerned breeding management and ethical considerations when compared to dogs ${ }^{11}$, ferrets ${ }^{12}$ or sheep ${ }^{13}$. Moreover, their pulpal, periapical and healing response is comparable to that of humans ${ }^{17,18}$.

Given we used rats of a very young age in our experimental model, we waited for them to be weaned - at the age of 21 days. Even though mouth opening at this age is still limited, the mandibular first molars are indeed fully erupted and present short roots and wide open apexes. Based on recent observations ${ }^{14,18}$ that pointed out that the development of the mandibular first molar is still incomplete by the age of 35 days old, younger rats were chosen for this model - i.e. four days post-weaning (25 days old $(\mathrm{Ci}))$ - so as to guarantee roots were still immature but access was sufficient for rotary instruments to expose dental pulp and cause its necrosis by leaving it open to the oral environment. Unfortunately, working with small animals in vivo does not permit to take intraoperative intraoral radiographs that would allow to take measurements and follow up the evolution and response of hard tissues to treatment thus making it necessary to await dissection of the hemimandibles and obtention of post-mortem radiographs and histological sections in order to be able to analyse results.

Following complete necrosis of the dental pulp, root development was halted in the group that received no further treatment (E1); the result was a wide root canal with thin walls and no further lengthening of the root which occurred as a consequence of the death of odontoblasts and cells of HERS, respectively.

In the experimental group that received treatment (E2), a regenerative endodontic therapy model that adhered to the principles applied to treating immature permanent human teeth was followed. For this purpose, newly-weaned rats with experimentally-induced necrosis were used and the regenerative procedure was carried in two steps (disinfection and blood-clot formation).

It is worth mentioning that procedures related to the regenerative protocol, including initial debridement of the root canal, placement of the bi-antibiotic paste and coronal seal could be carried out without major inconveniences even in spite of the reduced dimensions with which we were set to work. Likewise, the distal root was accessed again two weeks after to induce bleeding and formation of the intracanal blood clot and to place MTA upon the root canal orifice. The biggest challenge was perhaps encountered when manipulating MTA given the presence of the blood clot, the shallowness of the pulp chamber and the consistency of the material making both ability and practice necessary to achieve a coronal seal that remained in place until the moment of euthanasia.

For a number of authors ${ }^{5-7}$, the results following regenerative endodontics can be explained by the mobilisation of undifferentiated stem cells from the apical region which in combination with growth factors supplied by the bleeding are fundamental to trigger tissue regeneration. In other words, the granulation tissue presents in the apical region as a response to pulp necrosis acts as a potential niche and reservoir for regenerative processes. 
In the present model, complete development of the root was not achieved, but we were still able to observe an increase in the length of the root and the thickness of its walls therefore suggesting that the cells that were observed lining the walls of the root canal could be responsible for the synthesis and mineralisation of mineralised radicular tissues. Moreover, after performing regenerative endodontic procedures, histological characteristics of the periapical tissues were analysed observing a decrease in the periodontal ligament width with reduced inflammatory infiltrate and fewer osteoclasts per mm of bone surface. Even osteoblasts were identified on the surface of the surrounding alveolar bone.

Nonetheless, there is still much to be unravelled in regenerative endodontics, whether it is developing new substances for root canal disinfection that are safer than antibiotics or irrigants that might attract certain cell populations or using scaffolds for more biomimetic approaches ${ }^{19}$.

In conclusion, in our newly-weaned Wistar rat model we demonstrated that after experimental induction of pulp necrosis and root development arrest, an increase in length of the distal root and decrease in the apical diameter of its root canal were achieved by means of REPs. Therefore, the present model of pulp necrosis, canal disinfection using a bi-antibiotic paste and formation of an intracanal blood clot, is suitable and reproducible in order to study an analyse regenerative processes. It would be interesting to continue our work with immunohistochemical determinations to determine the kind of cells involved in the process and the tissues formed within the root canal.

\section{Ethics}

The ethics committee of the School of Dentistry of the University of Buenos Aires approval with the reference number (CD N012/2016 CICUAL).

\section{Acknowledgments}

This work was supported by project UBACYT: 20720150200012BA. The authors are grateful to Carlos Bárcenas for his expert assistance in animal care and in handling animals during procedures, Laboratory Technicians Mariela Lacave and Paula Rocha for processing the samples and obtaining the histological sections, and Sharon R. Oyhanart for kindly reviewing the manuscript.

\section{Conflicts Of Interest}

The authors declare no conflict of interest.

\section{References}

1. American Association of Endodontists. Glossary of endodontic terms. 10 th ed. Chicago: AAE; 2019.

2. Ostby BN. The role of the blood clot in endodontic therapy. An experimental histologic study. Acta Odontol Scand. 1961 Dec;19:324-53.

3. Hargreaves KM, Diogenes A, Teixeira FB. Treatment options: biological basis of regenerative endodontic procedures. J Endod. 2013 Mar;39(3 Suppl):S30-43. doi: 10.1016/j.joen.2012.11.025. 
4. Diogenes A, Ruparel NB, Shiloah Y, Hargreaves KM. Regenerative endodontics: a way forward. J Am Dent Assoc. 2016 May;147(5):372-80. doi: 10.1016/j.adaj.2016.01.009.

5. Huang GT, Sonoyama W, Liu Y, Liu H, Wang S, Shi S. The hidden treasure in apical papilla: the potential role in pulp/dentin regeneration and bioroot engineering. J Endod. 2008 Jun;34(6):645-51. doi: 10.1016/j.joen.2008.03.001.

6. Huang GT. Apexification: the beginning of its end. Int Endod J. 2009 Oct;42(10):855-66. doi: 10.1111/j.1365-2591.2009.01577.x.

7. Nosrat A, Kolahdouzan A, Hosseini F, Mehrizi EA, Verma P, Torabinejad M. Histologic outcomes of uninfected human immature teeth treated with regenerative endodontics: 2 case reports. J Endod. 2015 Oct;41(10):1725-9. doi: 10.1016/j.joen.2015.05.004.

8. Huang GT, Garcia-Godoy F. Missing concepts in de novo pulp regeneration. J Dent Res. 2014 Aug;93(8):717-24. doi: 10.1177/0022034514537829.

9. Alexander A, Torabinejad M, Vahdati SA, Nosrat A, Verma P, Grandhi A, et al. Regenerative endodontic treatment in immature noninfected ferret teeth using blood clot or synoss putty as scaffolds. J Endod. 2020 Feb;46(2):209-15. doi: 10.1016/j.joen.2019.10.029.

10. Pulyodan MK, Paramel Mohan S, Valsan D, Divakar N, Moyin S, Thayyil S. Regenerative endodontics: a paradigm shift in clinical endodontics. J Pharm Bioallied Sci. 2020 Aug;12(Suppl 1):S20-6. doi: 10.4103/jpbs.JPBS_112_20.

11. Stambolsky C, Rodríguez-Benítez S, Gutiérrez-Pérez JL, Torres-Lagares D, Martín-González J, Segura-Egea JJ. Histologic characterization of regenerated tissues after pulp revascularization of immature dog teeth with apical periodontitis using tri-antibiotic paste and platelet-rich plasma. Arch Oral Biol. 2016 Nov;71:122-8. doi: 10.1016/j.archoralbio.2016.07.007.

12. Torabinejad M, Corr R, Buhrley M, Wright K, Shabahang S. An animal model to study regenerative endodontics. J Endod. 2011 Feb;37(2):197-202. doi: 10.1016/j.joen.2010.10.011.

13. Altaii M, Cathro P, Broberg M, Richards L. Endodontic regeneration and tooth revitalization in immature infected sheep teeth. Int Endod J. 2017 May;50(5):480-91. doi: 10.1111/iej.12645.

14. Oyhanart SR, Canzobre MC. Methodological considerations for a model of endodontic treatment in Wistar rats. Acta Odontol Latinoam. 2020 Dec;33(3):153-64.

15. Thibodeau B, Trope M. Pulp revascularization of a necrotic infected immature permanent tooth: case report and review of the literature. Pediatr Dent. 2007 Jan-Feb;29(1):47-50.

16. Hameed MH, Gul M, Ghafoor R, Badar SB. Management of immature necrotic permanent teeth with regenerative endodontic procedures - a review of literature. J Pak Med Assoc. 2019 Oct;69(10):1514-20.

17. Scarparo RK, Dondoni L, Böttcher DE, Grecca FS, Rockenbach MI, Batista EL Jr. Response to intracanal medication in immature teeth with pulp necrosis: an experimental model in rat molars. J Endod. 2011 Aug;37(8):1069-73. doi: 10.1016/j.joen.2011.05.014.

18. Yoneda N, Noiri Y, Matsui S, Kuremoto K, Maezono H, Ishimoto T, et al. Development of a root canal treatment model in the rat. Sci Rep. 2017 Jun;7(1):3315. doi: 10.1038/s41598-017-03628-6.

19. Kaushik SN, Kim B, Walma AM, Choi SC, Wu H, Mao JJ, et al. Biomimetic microenvironments for regenerative endodontics. Biomater Res. 2016 Jun;20:14. doi: 10.1186/s40824-016-0061-7. 